\title{
The proper study of mankind
}

\author{
William M. Nauseef
}

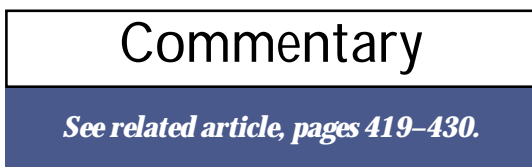

Inflammation Program and D epartment of M edicine, University of I owa and Veterans Administration M edical Center, Iowa City, lowa 52242, USA. Phone: (319) 356-1739; Fax: (319) 356-4600; E-mail: william-nauseef@uiowa.edu.

Approximately 30 years ago Klebanoff demonstrated the importance of myeloperoxidase (M PO) in the oxygendependent microbicidal activity of human phagocytes (1). The ability of M PO to catalyzethe oxidation of chloride and to chlorinate substrates $(2,3)$, properties unique among members of the protein family of animal peroxidases (4), likely ref lects the unusual linkage of its heme to the apoprotein (5). This capacity underlies its potent antimicrobial activity, since $\mathrm{HOCl}$ represents the proximal agent generated by the combined efforts of M PO and the products of the NADPH-dependent oxidase released during stimulation of phagocytes (6). H owever, the significance of peroxidase and $\mathrm{H}_{2} \mathrm{O}_{2}$ in the modification of biological substrates extends well beyond damaging invading microorganisms and includes processes as seemingly divergent as thesynthesis of thyroid hormone (7) and therespiratory burst seen during fertilization of sea urchin eggs (8). The capacity of the M PO- $\mathrm{H}_{2} \mathrm{O}_{2}$-halide system to modify proteins, lipids, and nucleic acids in a wide variety of biological contexts has been the subject of extensive study (9). Several laboratories have established that the MPO- $\mathrm{H}_{2} \mathrm{O}_{2}$-halide system, either as reconstituted components in vitro or as the result of phagocytestimulation, can oxidize lipoproteins and thereby generate atherogenic species. Such a formulation fits well with the concept of atherosclerosis as a chronic inflammatory disease (10), reflecting arterial damage induced by lipoproteins modified by the MPO- $-\mathrm{H}_{2} \mathrm{O}_{2}-$ halide system.

The H einecke lab (11) published, in the $\mathrm{Jl}$, the first evidence that linked M PO-dependent oxidation of lipids and lipoproteins to the pathogenesis of atherosclerosis. Detergent extracts of atherosclerotic human vessels contain proteins immunochemically related to MPO and exhibit both peroxidative and chlorinating activities, biochemical properties unique to $M P O$. Sensitive and quantitative assays for M PO-derived modifications of resident proteins from subintimal plaques (12-14) have since provided compelling evidence that peroxidatively active M PO is present in atherosclerotic lesions [reviewed in ref. 15]. M odifications such as the generation of chlorotyrosine (13) represent specific markers for M PO activity, whereas the detection of other species, such as the products of reactive aldehydes (16) constitute less incriminating evidence for a specific role for MPO. However, direct evidence that M POdependent events contribute to the formation or promotion of atherosclerosis was lacking, setting the stage for the report of Brennan et al. in this issue of the $\mathrm{Jl}$ (17).

As has been previously reported for an independently generated knockout of murine M PO (18), the mutants cre ated by Brennan et al. (17) have increased susceptibility to infection with Candida albicans. The murine knockout recapitulates the most dramatic immune defect seen in MPOdeficient humans, as neutrophils from affected individuals exhibit defective candidacidal activity in vitro (19), and several completely M PO-deficient patients have presented clinically with severe or fatal systemic candidiasis [reviewed in ref. 20]. To examine the influence of MPO activity on atherogenesis, Brennan et al. created a doubleknockout, crossing MPO-deficient mice with those that lack the receptor for LDL. Since the latter is a widely accepted murine model for human hypercholesterolemia (21) and atherosclerosis (22, 23), the authors reasoned that the double knockout would provide a powerful system to examine the contribution of MPO to atherogenesis. Accordingly onewould anticipate that the M PO-dependent effect would be manifested as a reduction in the amount or severity of atherosclerosis in the doubleknockout animals.

Two unexpected results emerge from their studies (17). First, animals that lack both the LDL receptor and M PO have more severe vasculopathy than do the animals with only LDL-receptor deficiency, a result contrary to that predicted if MPO were an important provocateur of atherosclerosis. However, even more unexpected was the second observation, that the atheromata in the positive control group, namely the LDL-receptor knockout animals, lack any trace of MPO. Neither immunochemical evidence of MPOrelated proteins nor M PO-specific posttranslational modifications of endoge nous substrates (e.g., chlorotyrosine) were detected in thelesions of the LD Lreceptor knockout mice expressing M PO in their granulocytes. The reader

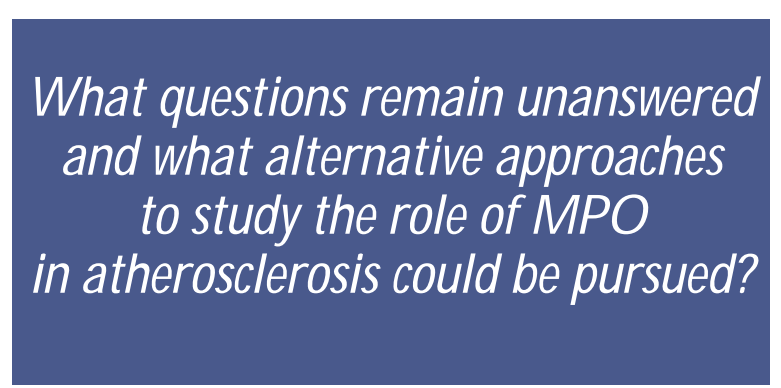

is left with the inescapable conclusion that, at least according to the premise underlying the fundamental question addressed by the authors, the disease produced in the LDL-receptor knockout mice did not mirror human atherosclerosis. In this light, can onedraw any conclusions from the data presented? What questions remain unanswered and what alternative approaches to study the role of MPO in atherosclerosis could be pursued?

First, the authors should be commended for therigor and integrity with which they assessed how accurately their animal model mirrored human disease, looking beyond simply reproducing its histologic features (17). 
Wholesaleinvestment in knockout animal models before rigorously assessing how faithfully they model the human situation can contribute to conclusions unrelated to human disease and propagate unnecessary subsequent studies. This may be especially true when characterizing phenomena in which phagocytic cells play a prominent role. Murine and human phagocytes havesignificant differences, some of which may undermine thefidelity of the murine system to model human disorders. The granules of murineneutrophils contain less $\beta$-glucuronidase, lysozyme, and alkaline phosphatase than do their human counterpart (24) and are devoid of bactericidal permeability-increasing protein or defensins (25), prominent contributors to human granulocyte antimicrobial defense. Furthermore, and especially pertinent to the study under discussion, murine neutrophils possess only $10 \%(26)$ to $20 \%(24)$ of the M PO present in human cells. Complicating further any interpretation of the functional implications of these interspecies differences in M PO content are the complex interactions of M PO and nitric oxide. Substantial evidence suggests that MPO-generated reactivenitrogen intermediates (RN IS) contribute to the nitration of LDL in human atherosclerotic lesions (27-30), that nitric oxide modulates the activity of M PO (31), and that murine phagocytes generate significantly greater amounts of RNIs than do human phagocytes $(32,33)$.

Second, the more extensive disease seen in MPO-deficient mice is a reminder that even in chronic inflammation, not all participants are proinflammatory. In fact, various elements may contribute to anti-inflammatory events or participatein termination of the inflammatory reaction. An example pertinent to this study is the compelling, al beit incomplete, suggestion that the $\mathrm{MPO}-\mathrm{H}_{2} \mathrm{O}_{2}$-halide system contributes to termination of activity of the NADPH oxidase, reported two decades ago in the $\mathrm{JI}$ (34). Consistent with this potential mechanism is the long-standing observation that monocytes and neutrophils from M PO-deficient individuals exhibit a prolonged respiratory burst $(35,36)$, suggesting that thenormal termination event(s) is delayed or defective in the absence of MPO. Perhaps the MPO detected in human atheromata contributes to the cessation of atherogenic events rather than promotes the disease.

Third, the sources of the two putative causative agents, namely M PO and oxidants, merit revisiting. The obvious candidate for reactive oxygen species, namely the phagocyte N APD H oxidase (37), may not contribute to oxidant generation at the site of vascular damage. Although subject to all the caveats raised earlier, recent data demonstrate that mice lacking a functional N AD PH -dependent phagocyte are not spared atherosclerosis when fed a high-cholesterol diet or bred with a strain deficient in apoE (38). Thus, the source of oxidants in vascular tissue may not be the NADPH oxidase of phagocytes but may be a member of the recently described family of NADPH oxidase homologues, called Nox (NADPH oxidases) in a recent review (39). N ox-1 is expressed in vascular tissue and could bethe source of low levels of oxidants sufficient to participate in lipid peroxidation.

The source of M PO is likewise uncertain. N ormally, mature M PO resides in the azurophilic granules of polymorphonuclear leukocytes and monocytes (40), its synthesis restricted to the promyel ocytic stage of myeloid development $(41,42)$. The transition from monocyte to macrophage is associated with a decrease in peroxidase activity (43), thought to reflect a loss in cellassociated MPO during differentiation into macrophages. Although the M PO detected within plaques could be the remnants of enzyme released during migration of phagocytes through the lesion early in the genesis of the plaque, neutrophils are not seen in atheromata and monocytes have relatively little MPO. An alternative, and testable, hypothesis is that tissue macrophages, within the context of theuniquemix of cytokines present in the atheromatous plaque subintimally, reinitiate transcription of the M PO gene. In contrast to bone marrow promyelocytes, foam cells do not possess azurophilic granules and thus would lack access to the subcellular compartment for storage of M PO. Without an intracellular target and/or targeting mechanism, the newly synthesized MPO precursor in foam cells would enter thesecretory pathway and be released into the extracellular space. Were this mechanism operative, immunochemical analysis of such lesions would detect the 90-kDa M PO precursor rather than processed subunits of mature lysosomal MPO.

Lastly, in light of the limitations of the model, the best test of the role of M PO in human atherosclerosis should comefrom clinical studies. Of course, the least ambiguous study would compare the prevalence or risk of atherosclerosis in individuals with and without M PO. Although complete M PO deficiency is relatively common [reviewed in ref. 20], a large population study would be necessary to provide sufficient numbers to support firm conclusions. Alternatively, one could determine whether the amount of functional MPO within granulocytes correlated with a graded risk of atherosclerosis, analogous to the recently described relationship between factor VII levels and risk for myocardial infarction (44). The promoter region of the M PO gene has an allelic polymorphism wherein the presence of $G$ or $\mathrm{A}$ at nucleotide- 463 effects 25 -fold differences in its transcription (45). This polymorphism is likely reflected in differential levels of M PO protein and activity within circulating granulocytes, affording investigators an accessible means to assess M PO levels and correlate them with the presence of atherosclerotic heart disease.

In summary, the study by Brennan et al. (17), likemany excellent studies, raises more questions than it answers, many of which extend well beyond the focus of the initial issue addressed. M ost striking is the reminder that the synergy of phagocyteantimicrobial elements, often synonymous with components of inflammation, may complicate theinterpretation of murine knockout studies, as el egantly discussed recently by $N$ athan and Shiloh (33). Despite their utility as powerful analytical tools, perhaps we should temper enthusiasm for this experimental approach with Alexander Pope's admonition from Epistle II of An Essay on Man, "The proper study of mankind is man."

\section{Acknowledgments}

Work in theN auseef lab is supported by grants from the US Public Health Service (Al-34879, HL-53592, Al-44642) and a M erit Review Grant from the Veterans Administration.

\footnotetext{
1. Klebanoff, S.J. 1970. M yeloperoxidase: contribution to the microbicidal activity of intact leukocytes. Science. 169:1095-1097.
} 
2. Weiss, S.J., Klein, R., Slivka, A., and Wei, M. 1982. Chlorination of taurine by human neutrophils. Evidencefor hypochlorous acid generation. J. Clin. Invest. 70:598-607.

3. Foote, C.S., Goyne, T.E., and Lehrer, R.I. 1983. Assessment of chlorination by human neutrophils. Nature. 301:715-726.

4. Ferrari, R.P. 2000. Structure function relationships amongst members of the animal peroxidase family of proteins. In The peroxidase multigenefamily of enzymes: biochemical basisand clinical applications. P.E. Petrides and W.M . N auseef, editors. Springer-Verlag. Berlin, Germany. 114-121.

5. Kooter, I.M ., et al. 1999. Thesulfonium ion linkage in myeloperoxidase. J. Biol. Chem. 274:26794-26802.

6. Winterbourn, C.C., Van Dalen, C.J., Hampton, M.B., and Kettle, A.J. 2000. Reactions of myeloperoxidase and production of hypochlorous acid in neutrophil phagosomes. In The peroxidasemultigenefamily of enzymes: biochemical basis and clinical applications. P.E. Petrides and W.M . N auseef, editors. Springer-Verlag. Berlin, Germany. 58-67.

7. de Vijlder, J.J.M., and Bikker, H. 2000. Biochemistry and physiology of thyroid peroxidase. In The peroxidase multigene family of enzymes: biochemical basisand clinical applications. P.E. Petrides and W.M. Nauseef, editors. Springer-Verlag. Berlin, Germany. 129-132.

8. H einecke, J.W., and Shapiro, B.M . 1989. Respiratory burst oxidase of fertilization. Proc. $N$ atl. Acad. Sci. USA. 86:1259-1263.

9.Thivierge, M., Parent, J.-L., Stankova, J., and Rola-Pleszczynski, M. 1999. Modulation of formyl peptide receptor expression by IL-10 in human monocytes and neutrophils. J. I mmunol. 162:3590-3595.

10. Ross, R. 1999. Atherosclerosis: an inflammatory disease. N. Engl. J. M ed. 340:115-126.

11. D augherty, A., D unn, J.L., Rateri, D .L., and Heinecke, J.W. 1994. M yeloperoxidase, a catalyst for lipoprotein oxidation, is expressed in human atherosclerotic lesions. J. Clin. Invest. 94:437-444.

12. Hazell, L.J., et al. 1996. Presence of hypochlorite modified proteins in human atherosclerotic lesions. J. Clin. Invest. 97:1535-1544.

13. H azen, S.L., and H einecke, J.W. 1997. 3-chlorotyrosine, a specific marker of myel operoxidase-catalyzed oxidation, is markedly elevated in low density lipoprotein isolated from human atherosclerotic intima. J. Clin. Invest. 99:2075-2081.

14. Malle, E., et al. 2000. Immunohistochemical evidencefor the myeloperoxidase $/ \mathrm{H}_{2} \mathrm{O}_{2}$ / halidesystem in human atherosclerotic lesions. Colocalization of myeloperoxidase and hypochlorite-modified proteins. Eur. J. Biochem. 267:4495-4503.

15. Podrez, E.A., Abu-Soud, H.M., and Hazen, S.L. 2000. M yeloperoxidase-generated oxidants and atherosclerosis. FreeRadic. Biol. M ed. 28:1717-1725.
16. Anderson, M.M., Hazen, S.L., H su, F.F., and Heinecke, J.W. 1997. Human neutrophils employ the myeloperoxidase-hydrogen peroxide-chloride system to convert hydroxy-amino acids into glycolaldehyde, 2-hydroxypropanal, and acrolein. J. Clin. Invest. 99:424-432.

17. Brennan, M.-L., et al. 2001. Increased atherosclerosis in myeloperoxidase-deficient mice. J. Clin. Invest. 107:419-430.

18. Aratani, Y., et al. 1999. Severe impairment in early host defense against Candida albicans in mice deficient in myeloperoxidase. Infect. Immun. 67:1828-1836.

19. Lehrer, R.I., and Cline, M.J. 1969. Leukocyte myeloperoxidase deficiency and disseminated candidiasis: the role of myeloperoxidase in resistance to Candida infection. J. Clin. Invest. 48:1478-1488.

20. Dinauer, M.C., N auseef, W.M., and N ewburger, P.E. 2001. Inherited disorders of phagocyte killing. In The metabolic and molecular bases of inherited diseases. C.R. Scriver et al., editors. McGraw-Hill. New York, New York, USA. 4857-4887.

21. Ishibashi, S., et al. 1993. Hypercholesterolemia in low density lipoprotein receptor knockout mice and its reversal by adenovirus-mediated gene delivery. J. Clin. Invest. 92:883-893.

22. Tangirla, R.K., Rubin, E.M., and Palinski, W. 1995. Quantitation of atherosclerosis in murine models: correlation between lesions in the aortic origin and in the entire aorta, and differences in the extent of lesions between sexes in LDL receptor-deficient and apolipoprotein E-deficient mice. J. Lipid Res. 36:2320-2328.

23. Witztum, J.L. 2000. M urine models for study of lipoprotein metabolism and atherosclerosis. J. Clin. Invest. 92:536-537.

24. Rausch, P.G., and Moore, T.G. 1975. Granule enzymes of polymorphonuclear neutrophils: a phylogenetic comparison. Blood. 46:913-919.

25. Elsbach, P., Weiss, J., and Levy, O. 1999. Oxygenindependent antimicrobial systems of phagocytes. Lip pincott Williams \& Wilkins. Baltimore, M aryland, USA. 801-817.

26. N oguchi, N., et al. 2000. Role of myeloperoxidasein theneutrophil-induced oxidation of low density lipoprotein as studied by myeloperoxidase-knockout mouse. J. Biochem. 127:971-976.

27. Podrez, E.A., Schmitt, D., H off, H.F., and Hazen, S.L. 1999. M yeloperoxidase-generated reactive nitrogen species convert LDL into an atherogenic form in vitro. J. Clin. Invest. 103:1547-1560.

28. Podrez, E.A., et al. 2000. The macrophage scavenger receptor $C D 36$ is the major receptor for LDL recognition following modification by monocyte-generated reactive nitrogen species. J. Clin. Invest. 105:1095-1108.

29. Hazen, S.L., et al. 1999. Formation of nitric oxide-derived oxidants by myeloperoxidase in monocytes. Pathways for monocyte-mediated protein nitration and lipid peroxidation in vivo.
Circ. Res. 85:950-958.

30. Eiserich, J.P., et al. 1998. Formation of nitric oxide-derived inflammatory oxidants by myeloperoxidase in neutrophils. Nature. 391:393-397.

31. Abu-Soud, H.M., and H azen, S.L. 2000. Nitric oxide modulates the catalytic activity of myeloperoxidase. J. Biol. Chem. 275:5425-5430.

32. M acM icking, J., Xie, Q.-W., and N athan, C. 1997. $\mathrm{N}$ itric oxide and macrophage function. Annu. Rev. Immunol. 15:323-350.

33. Nathan, C., and Shiloh, M. 2000. Reactive oxygen and nitrogen intermediates in the relationship between mammalian hosts and microbial pathogens. Proc. Natl. Acad. Sci. USA. 97:8841-8848.

34. Jandl, R.C., et al. 1978. Termination of the respiratory burst in human neutrophils. J. Clin. Invest. 61:1176-1185.

35. Rosen, H., and Klebanoff, S.J. 1976. Chemiluminescence and superoxide production by myeloperoxidase-deficient leukocytes. J. Clin. Invest. 58:50-60.

36. N au seef, W.M ., M etcalf, J.A., and Root, R.K. 1983. Role of myeloperoxidase in the respiratory burst of human neutrophils. Blood. 61:483-491.

37. Babior, B.M. 1999. NADPH oxidase: an update. Blood. 93:1464-1476.

38. Kirk, E.A., et al. 2000. Impaired superoxide production due to a deficiency in phagocyte NADPH oxidase fails to inhibit atherosclerosis in mice. Arterioscler. Thromb. Vasc. Biol. 20:1529-1535

39. Lambeth, J.D., Cheng, G., Arnold, R.S., and Edens, W.A. 2000. N ovel homologs of gp91phox. Trends Biochem. Sci. 25:459-461.

40. Bainton, D.F., Ullyot, J.L., and Farquhar, M.G 1971. The development of neutrophilic polymorphonuclear leukocytes in human bonemarrow. Origin and content of azurophil and spe cific granules. J. Exp. M ed. 134:907-934.

41. N auseef, W.M., Olsson, I., and StrömbergArnljots, K. 1988. Biosynthesis and processing of myeloperoxidase: a marker for myeloid differentiation. Eur. J. H aematol. 40:97-110.

42. Gullberg, U., Andersson, E., Garwicz, D., Lindmark, A., and Olsson, I. 1997. Biosynthesis, processing and sorting of neutrophil proteins: insight into neutrophil granule development. Eur. J. H aematol. 58:137-153.

43. N akagawara, A., N athan, C.F., and Cohn, Z.A. 1981. Hydrogen peroxidemetabolism in human monocytes during differentiation in vitro. J. Clin. Invest. 68:1243-1252.

44. Girelli, D., et al. 2000. Polymorphisms in thefactor VII gene and the risk of myocardial infarction in patients with coronary artery disease. $\mathrm{N}$. Engl. J. M ed. 343:774-780.

45. Piedrafita, F.J., et al. 1996. An Alu element in the myel operoxidase promoter contains a compositeSP1-thyroid hormoneretinoic acid response element. J. Biol. Chem. 271:14412-14420. 